\title{
UV-Vis Spectra-Activated Droplet Sorting for Label-Free Chemical Identification and Collection of Droplets
}

\section{Journal Article}

Author(s):

Duncombe, Todd A.; Ponti, Aaron (D); Seebeck, Florian P.; Dittrich, Petra S. (D)

Publication date:

2021-09-28

Permanent link:

https://doi.org/10.3929/ethz-b-000509599

\section{Rights / license:}

Creative Commons Attribution-NonCommercial-NoDerivatives 4.0 International

Originally published in:

Analytical Chemistry 93(38), https://doi.org/10.1021/acs.analchem.1c02822 


\title{
UV-Vis Spectra-Activated Droplet Sorting for Label-Free Chemical Identification and Collection of Droplets
}

\author{
Todd A. Duncombe, Aaron Ponti, Florian P. Seebeck, and Petra S. Dittrich*
}

Cite This: Anal. Chem. 2021, 93, 13008-13013

Read Online

ACCESS

Llll Metrics \& More

Article Recommendations

Supporting Information

ABSTRACT: We introduce the UV-vis spectra-activated droplet sorter (UVADS) for high-throughput label-free chemical identification and enzyme screening. In contrast to previous absorbancebased droplet sorters that relied on single-wavelength absorbance in the visible range, our platform collects full UV-vis spectra from 200 to $1050 \mathrm{~nm}$ at up to 2100 spectra per second. Our custombuilt open-source software application, "SpectraSorter," enables real-time data processing, analysis, visualization, and selection of droplets for sorting with any set of UV-vis spectral features. An optimized UV-vis detection region extended the absorbance path length for droplets and allowed for the direct protein quantification down to $10 \mu \mathrm{M}$ of bovine serum albumin at $280 \mathrm{~nm}$. UV-vis spectral data can distinguish a variety of different chemicals or

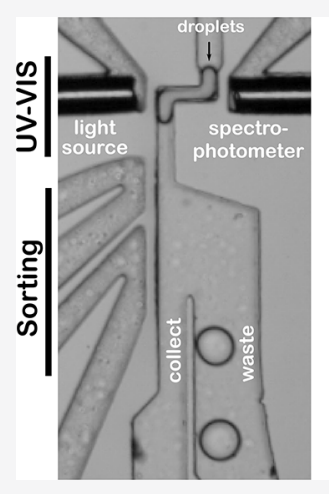
full-spectrum in-droplet UV-VIS spurious events (such as air bubbles) that are inaccessible at a single wavelength. The platform is used to measure ergothionase enzyme activity from monoclonal microcolonies isolated in droplets. In a label-free manner, we directly measure the ergothioneine substrate to thiourocanic acid product conversion while tracking the microcolony formation. UVADS represents an important new tool for high-throughput label-free in-droplet chemical analysis.

$\mathrm{D}$ roplet microfluidic platforms leverage rapidly generated sub-nanoliter discretized reactors and fast reaction times to achieve high-throughput and massively parallel analytics. ${ }^{1}$ When real-time readouts are combined with active microfluidic components-such as electrodes or actuators-for on-demand droplet sorting, ${ }^{2-6}$ the platform can be applied to a diverse set of screening applications, from directed evolution ${ }^{7-9}$ and single-cell analysis ${ }^{10-14}$ to chemical synthesis and particle analysis. ${ }^{15}$ Bright-field and fluorescence microscopies are the most common tools for real-time readouts used due to their compatibility with transparent materials of glass and polymer microfluidic chips. The confined nature of microdevices and the minuscule sample volumes make alternative readouts challenging. As a result, the majority of microfluidic screening applications to date revolve around assays using conventional microscopes. ${ }^{16,17}$ While this has proven powerful across multiple applications, in others, where fluorescent labels and standard microscopy are incompatible, droplet microfluidics has traditionally been limited.

To address this bottleneck, there has been tremendous progress in recent years in integrating droplet microfluidics with a number of label-free analytical measurements. ${ }^{6,8,14,18--24,25-27}$ Notably, Holland-Moritz et al. ${ }^{22}$ developed a mass spectrometry-activated droplet sorter that circumvents the sample-destructive nature of mass spectrometry by splitting droplets into two streams, analyzing a portion with electrospray ionization mass spectrometry in real-time, and sorting the partner droplet stream based on the mass spectra. While a very promising approach, its overall throughput of 0.7 samples per second leaves much to be desired for high-throughput screens. Using the nondestructive label-free readout of Raman spectroscopy, Wang et al. ${ }^{6,21}$ demonstrated Raman-activated droplet sorting at up to 4.3 cells per second. While powerful, their Raman measurement is performed in-solution prior to droplet formation, which precludes it from some screening applications. Given the great potential in label-free droplet sorter approaches, we expect to see significant investments and improvements in the near future.

A common benchtop label-free quantitation readout is UVvis spectroscopy, which interrogates molecular structures directly by chemical absorbance of incident light over a large spectrum, for example, from 200 to $1000 \mathrm{~nm}$. Analytically, this is particularly useful at UVB and UVC wavelengths $(<315$ $\mathrm{nm}$ ), where DNA, aromatic amino acids, and other unique chemical signatures can be observed. ${ }^{28}$ There have been

Received: July 6, 2021

Published: September 17, 2021 
several published microfluidic devices that utilize droplet absorbance measurements ${ }^{23,29,30}$ but none, to date, that incorporate high-throughput full-spectrum UV-vis spectroscopy in screening assays. Gielen et al. ${ }^{8}$ developed an absorbance-activated droplet sorter for the use of colorimetric enzymatic assays with integrated optical fibers, a singlewavelength visible-range LED light source, and a photodiode. Their platform was well-suited for high-throughput droplet assays for directed evolution but was restricted to assays compatible with a single wavelength readout.

In this paper, we introduce a UV-vis spectra-activated droplet sorter (UVADS, Figure 1). The platform performs

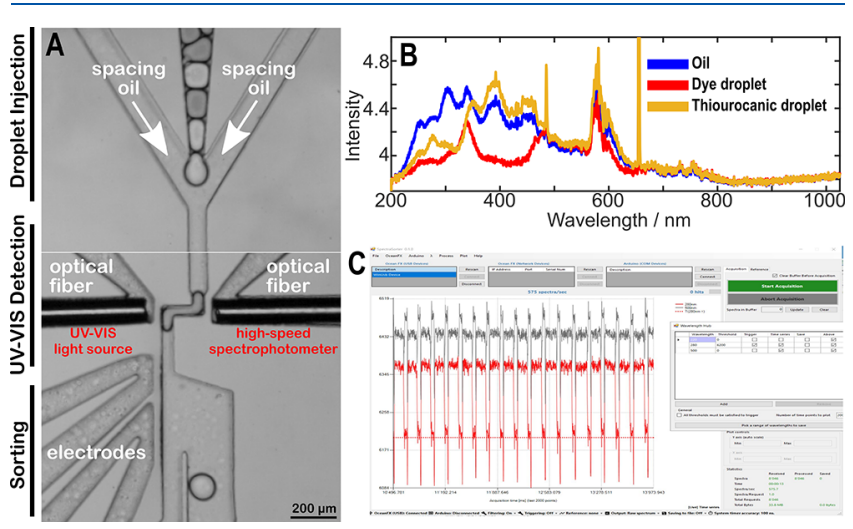

Figure 1. UVADS enables high-throughput label-free chemical identification in droplets and their on-demand collection. (A) Micrograph of the chip. The injected droplets are spaced by oil and flow past a detection region spanned by optical fibers and to a sorting region. Incident broadband $\mathrm{UV}$-vis light is absorbed by the intersecting oil/droplet emulsion and then (B) transmitted spectra are detected with a high-speed spectrophotometer at a speed of up to 2100 spectra per second. (C) Screenshot of our "SpectraSorter" software that processes droplet absorbance spectra in real-time and performs multiple-wavelength thresholding to select a droplet for sorting.

high-speed UV-vis spectroscopy from 200 to $1050 \mathrm{~nm}$ droplet absorption spectra-with $0.4 \mathrm{~nm}$ resolution-at up to 2100 spectra per second with a custom-developed and open-source software package. ${ }^{31}$ The real-time absorbance spectra processing and analysis is complete in several milliseconds, and droplet sorting can be performed on any single or multiple wavelengths, or over a spectrum. Using an optimized UV-vis detection region, our platform can directly measure unlabeled bovine serum albumin (BSA) down to $10 \mu \mathrm{M}$ in concentration. Furthermore, we demonstrate that the system can directly track and sort droplets by enzymatic activity for screening assays by directly measuring the conversion of native substrates to product in the deep UV range. UVADS is the first platform that combines high-throughput full-spectrum UV-vis in-droplet measurements, real-time spectral analysis, and subsequent sorting for multiple-wavelength features.

\section{EXPERIMENTAL SECTION}

UVADS Device Architecture. The polydimethylsiloxane (PDMS) microfluidic dielectrophoretic droplet sorter architecture used in this study, as depicted in Figure 1A, was directly adapted from a previously published sorter design ${ }^{8}$ with both a modified UV-vis detection region and a new twolayer PDMS architecture to accommodate larger UVcompatible optical fibers. Droplets are generated in a separate chip. Injected droplet spacing is controlled by two oil channels, which are independently manipulated from droplet injection using a separate syringe pump. Downstream of the spacing module, droplets enter the UV-vis detection region. Incident light from a broadband combined deuterium and halogen light source (DH-2000-BAL, Ocean Insight, Duiven, Netherlands) passes through optical fibers, $30 \mu \mathrm{m}$ PDMS channel walls, and a droplet, and the transmitted spectra are detected with a highspeed spectrophotometer (Figure 1B, OceanOptics FX, Ocean Insight).

The $105 \mu \mathrm{m}$-diameter UV-compatible optical fibers (Thorlabs, M111L02, Newton, NJ, USA) are inserted directly into PDMS channels to precisely align them adjacent to the droplet injection path. To both decouple the dimensions of the droplet channel (e.g., $40 \mu \mathrm{m}$ height) from the optical fiber channel (110 $\mu \mathrm{m}$ height) and to center the optical fiber along the droplet channel axis, our device utilized a two-step height SU8 mold and a two-layer PDMS device. Two symmetric molds were generated corresponding to the PDMS layers. They were aligned with channel features facing one another and covalently bound with an additional PDMS cross-linker. As expected, we found that the axially aligned configuration of the droplet channel to the optical fiber dramatically improved the UV-vis signal when droplet dimensions were significantly smaller than that of the optical fibers. After the UV-vis detection region, the channel expands and enters the sorting region-flanked by two electrode channels filled with of $3 \mathrm{M}$ $\mathrm{KCl}$. In the neutral state, droplets are slightly biased to flow to the waste channel based on expansion of the droplet after the constriction and the relative hydrodynamic resistances of the outlet channels. When the $\mathrm{KCl}$ electrodes are activated by 400 $\mathrm{V}$ at $1 \mathrm{kHz}$ (for $1-20 \mathrm{~ms}$ depending on the flow rates), the droplet is pulled toward the electrode and is collected in the collection channel. Additional details on SU8 mold fabrication, device assembly, image acquisition, and droplet generation are described in Supporting Information.

SpectraSorter Software Package. Real-time UV-vis droplet sorting is performed with our custom open-source software package, "SpectraSorter" (Figure 1C). SpectraSorter is a user-friendly software application compatible with Ocean Insight FX spectrophotometers. It allows for real-time data processing, visualization, thresholding for multiple-spectral features, and sorting to be triggered within milliseconds. The software is available for download online. ${ }^{31}$ The sorting trigger from SpectraSorter is sent via an Arduino Mega 2560 to a WaveStation 2022 function generator (Teledyne Lecroy, Chesnut Ridge, NY, USA) and TREK model PZD700A high-voltage power supply (model PZD700A, Trek Inc., Lockport, NY, USA). Typical droplet sorting is performed at droplet frequencies between 5 and $50 \mathrm{~Hz}$. The critical limitation is the requisite integration time for the spectrophotometer to acquire an adequate signal. A brighter light source could potentially allow for this to be reduced and droplet sorting speeds to be accelerated above $100 \mathrm{~Hz}$.

Sample Preparation. Reagents BSA (A2153, SigmaAldrich), ergothioneine (E7521, Sigma-Aldrich), or red dye (TRAWOSA, St. Gallen, Switzerland) were dissolved into DI water and filtered through a $0.2 \mu \mathrm{m}$ hydrophilic filter prior to use. Bacterial strains Escherichia coli K12 MG1655 $[\mathrm{pSEVA} 271-s f g f p]^{32}$ are used in Figure 4. Ergothionase isolated from Treponema denticola was previously expressed in E. coli using a modified vector pET19(+) vector, E. coli BL21 (DE3) [pET19-ergothionase $].^{33}$ In Figure 5, we tested the wild 
type and three strains containing amino acid point mutations Y54F, K384M, and E412Q (see previous work for details). ${ }^{33}$ Bacterial strains were seeded in antibiotic-free MuellerHinton media (70192, Sigma-Aldrich) in liquid culture for $30 \mathrm{~min}$ at $37^{\circ} \mathrm{C}$ at $220 \mathrm{rpm}$ prior to use. Bacterial cultures were diluted 1 to 10 times in additional media prior to the droplet encapsulation of single cells. In Figure 4, for GFPexpressing cells, the diluting media contained Mueller-Hinton media and $50 \mu \mathrm{g} / \mathrm{mL}$ kanamycin. In Figure 5, for ergothionase expression, the diluting media contained Mueller-Hinton media, $5 \mathrm{mM}$ ergothioneine, and $1 \mathrm{mM}$ isopropyl- $\beta$-Dthiogalactoside (IPTG, 10724815001, Sigma-Aldrich).

\section{RESULTS AND DISCUSSION}

Optimized Detection Region for Absorbance. While the small feature sizes of microfluidics hold numerous analytical advantages-such as reduced reagent consumption, rapid reactions, predictable mass transport, and a high surfaceto-volume ratio - the limited quantity of molecules and short path lengths of microreactors generate significant challenges for label-free readouts. Absorbance measurements are directly proportional to the absorbance path length. The UV-vis detection region is defined by the $105 \mu$ m-diameter cross section of the optical fibers and the incident fluidic channel. To maximize the absorbance measurement, it is necessary to position droplets along the axis of the optical fiber cross section such that the droplet fully occupies the detection region. We modified the UV-vis detection region with a rightangle turn and a radial constriction, resulting in droplets being stretched along the axis of the optical fibers-effectively increasing the path length of absorbance (Figure 1A).

To test the impact of this modification, we assessed various lengths (from 50 to $300 \mu \mathrm{m}$ ), channel widths, and heights, as detailed in Figure S1, for droplets containing BSA. As expected, when the entire detection region was filled, the design with the longest path length $(300 \mu \mathrm{m})$ had the highest absorbance measurement. In practice, we are limited by the working volume of the droplet and therefore calculated the volume-normalized absorbance for each design. We found that the maximum volume-normalized absorption had a $100 \mu \mathrm{m}$ length because diffractive losses as the spacing between optical fibers are increased. If different droplet volumes are desired, one can tune the fluidic channel height such that the detection region is fully occupied. As the fluidic channel height is defined in a separate fabrication step from the fiber optics channel, this is a fairly straightforward process.

Using these optimized settings, we quantified BSA label-free in droplets by its 236 and $280 \mathrm{~nm}$ absorption peaks for concentrations from 10 to $500 \mu \mathrm{M}$ (Figure $2 \mathrm{~B}$ ). To achieve the $10 \mu \mathrm{M}$ measurement, integration times had to be increased to $2 \mathrm{~ms}$, slowing down the operation of the sorter. The trade-off between sensitivity and speed is unavoidable in absorbance measurements and should be considered when designing an assay for this readout. We typically operated the UV-vis droplet sorter with reagent concentrations ranging between 0.1 and $10 \mathrm{mM}$ to allow for robust signals with acquisition times ranging between 0.5 and $2 \mathrm{~ms}$. To avoid aliasing, we aim to acquire at least four spectra per droplet which sets a throughput limit for our system. In contrast, fluorescent droplet sorters can operate with acquisition times less than 0.1 $\mathrm{ms}$ and can sort 10 times faster than UVADS.

High-Throughput Label-Free Chemical Detection. The multidimensional nature of UV-vis spectroscopy makes

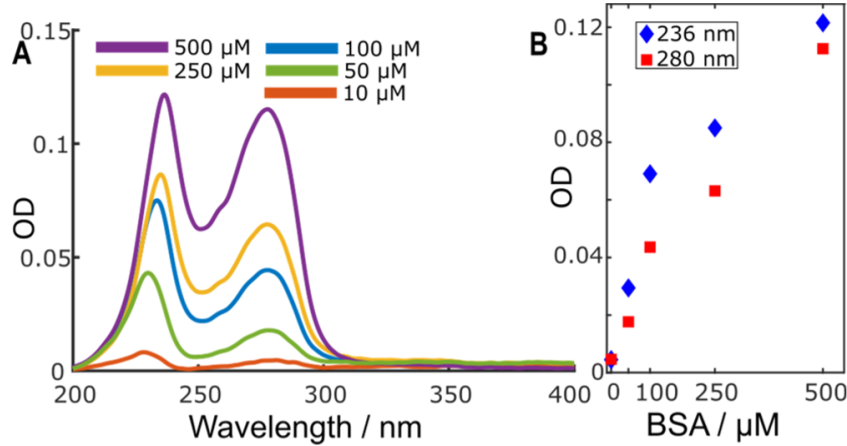

Figure 2. UV protein quantitation in-droplet of BSA-containing droplets. (A) Absorbance spectra and (B) calibration curve for BSA droplets from 10 to $500 \mu \mathrm{M}$ at 236 and $280 \mathrm{~nm}$, the UVC and UVB range, respectively. DI water droplets were used for the reference spectrum to calculate OD.

it well-suited for directly differentiating the contents of droplets in high-throughput label-free assays. In Figure 3, we demonstrate this by distinguishing the identity of over 12,700 droplets in less than a $7 \mathrm{~min}$ period by observing at multiple wavelengths along the UV-vis absorbance spectra. We used a $0.5 \mathrm{~ms}$ acquisition time to record approximately at 900 spectra per second with a syringe-controlled oil flow rate of $12 \mu \mathrm{L} / \mathrm{min}$ and a droplet rate of $4 \mu \mathrm{L} / \mathrm{min}$. In this demonstration, droplets contained either $5 \mathrm{mM}$ BSA, $5 \mathrm{mM}$ thiourocanic acid, or a red food-coloring dye. Each could be differentiated by their unique absorbance spectra signature. During high-throughput measurements for operational simplicity and to enable automated real-time correction for sensor drift via SpectraSorter, the reference spectrum of the fluorinated oil phase was used in the absorbance optical density (OD) calculations. As the oil phase has slightly lower optical transmittance than water, this resulted in negative ODs at some wavelengths.

In Figure 3A, the OD of the droplets at 280, 311, and 400 $\mathrm{nm}$ is plotted for a $7 \mathrm{~min}$ window. From these distinct chemical absorbance peaks, we can determine their contents label-free, as highlighted in Figure 3B in a $50 \mathrm{~ms}$ time window. A droplet containing thiourocanic acid had a peak at $311 \mathrm{~nm}$. A droplet containing BSA had a peak at $280 \mathrm{~nm}$. A droplet containing red dye had peaks at all three wavelengths. Furthermore, droplets that had merged could also be distinguished based on both wider peak widths and combined contents. In aggregate, in this experiment, we detected and identified the contents of droplets at over $30 \mathrm{~Hz}$, but the acquisition is capable of analysis at up to $100 \mathrm{~Hz}$ when incident droplets are optimally distributed, as seen in Figure 3B for the detection of 5 droplets in $50 \mathrm{~ms}$. A 3D absorbance scatter plot in Figure $3 \mathrm{C}$ highlights the multidimensional label-free chemical identification that this readout enables.

Monitoring Monoclonal Microcolony Growth. Among one of the most common uses of absorbance in a life science laboratory is to monitor cell growth label-free. Recent publications have demonstrated this in droplets using light scattering ${ }^{19}$ or capacitive sensing, ${ }^{24}$ but they lack the full spectral data that are typically acquired in a benchtop system. To assess cell-growth measurements in our in-droplet UV-vis platform, this GFP-expressing strain of E. coli K12 MG1655 [pSEVA271-sfgf $p]^{32}$ was used. Cells at an OD of 0.1 (at 600 $\mathrm{nm}$ measured with a benchtop system) were diluted into Mueller-Hinton cell media and then stochastically isolated in droplets using a standard flow-focusing junction. They were 

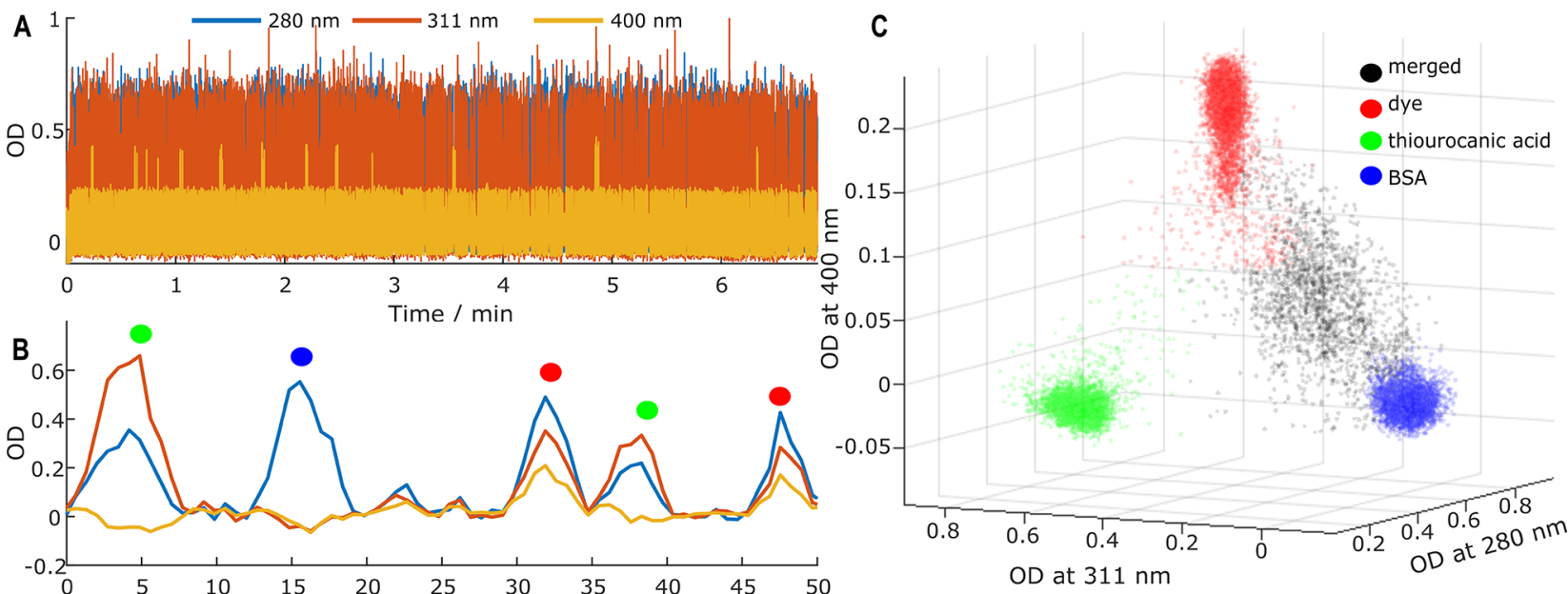

Figure 3. High-throughput label-free droplet identification via UV-vis spectroscopy. Droplets containing either $5 \mathrm{mM}$ thiourocanic acid, $5 \mathrm{mM}$ $\mathrm{BSA}$, or red food-coloring were generated in a separate flow-focusing device. (A) In-droplet UV-vis spectroscopy was used to analyze over $12.7 \mathrm{k}$ droplets in $7 \mathrm{~min}$ for an aggregate throughput of $\sim 30 \mathrm{~Hz}$. The OD at $280 \mathrm{~nm}$ (blue), $311 \mathrm{~nm}$ (red), and $400 \mathrm{~nm}$ (yellow) is plotted over time. (B) Zoomed-in $50 \mathrm{~ms}$ time window displays the high-throughput identification of five droplets with UV-vis spectroscopy. Droplet identities are indicated with blue circles: BSA (blue), dye (red), thiourocanic acid (green), and merged droplet (black). By the simultaneous assessment of absorbance at multiple wavelengths in the UV range, direct label-free droplet identification could be performed. (C) 3D scatter plot of droplet absorbance at 280,311 , and $400 \mathrm{~nm}$, with their corresponding labels.

incubated at $37{ }^{\circ} \mathrm{C}$ overnight and then analyzed with the UVADS. After incubation and cell growth, a distinct increase in $\mathrm{OD}$ is observed in E. coli-containing droplets, as shown in Figure 4A. This is plotted in Figure 4B for droplets as they serially pass the UV-vis detector for ODs at 280 and $600 \mathrm{~nm}$. The $280 \mathrm{~nm}$ wavelength is a standard wavelength used to monitor protein content by the presence of aromatic amino acids, while $600 \mathrm{~nm}$ is the standard wavelength to assess cell growth by light scattering. One can clearly distinguish $E$. coli microcolony-containing droplets by the $280 \mathrm{~nm}$ peak and the slight increase at $600 \mathrm{~nm}$ when compared to empty droplets. Furthermore, we can easily distinguish air bubbles that appear as high absorbance peaks across the UV-vis spectrum. By sorting for droplets containing an OD greater than 0 at $280 \mathrm{~nm}$ and less than 0.1 at $600 \mathrm{~nm}$ (to exclude bubbles), we collected droplets containing microcolonies. The initial and final droplet populations are displayed in Figure 4C. Sorting was observed to be highly accurate at $97.8 \%$, with errors occurring when injected droplet spacing was poor or there was debris in the system. As shown in Figure 4D, we interrogate the droplet population and identify the droplets in which cell growth occurred.

Label-Free Reaction Monitoring of Ergothionase Activity. The micronutrient ergothioneine, a thio-histidine betaine amino acid, has garnered significant interest as a potential therapeutic antioxidant, ${ }^{34,35}$ prompting researchers to investigate its metabolic lifecycle, from microbial biosynthesis to its degradative pathways. ${ }^{35}$ A recent publication studied its degradation via the enzyme ergothionase, from the bacterial oral pathogen $T$. denticola and expressed in E. coli. ${ }^{33}$ In the reaction, ergothionase catalyzes the 1,2-elimination of trimethylamine from ergothioneine to produce thiourocanic acid. No fluorescent probes exist to monitor the ergothionase reaction. In macroscale analysis, the readout is performed by benchtop UV-vis spectroscopy, as the production of thiourocanic acid results in a pronounced absorbance peak at $311 \mathrm{~nm}$ and a reduction of absorbance at $257 \mathrm{~nm}$ from the ergothioneine substrate. Previous single-wavelength absorb-
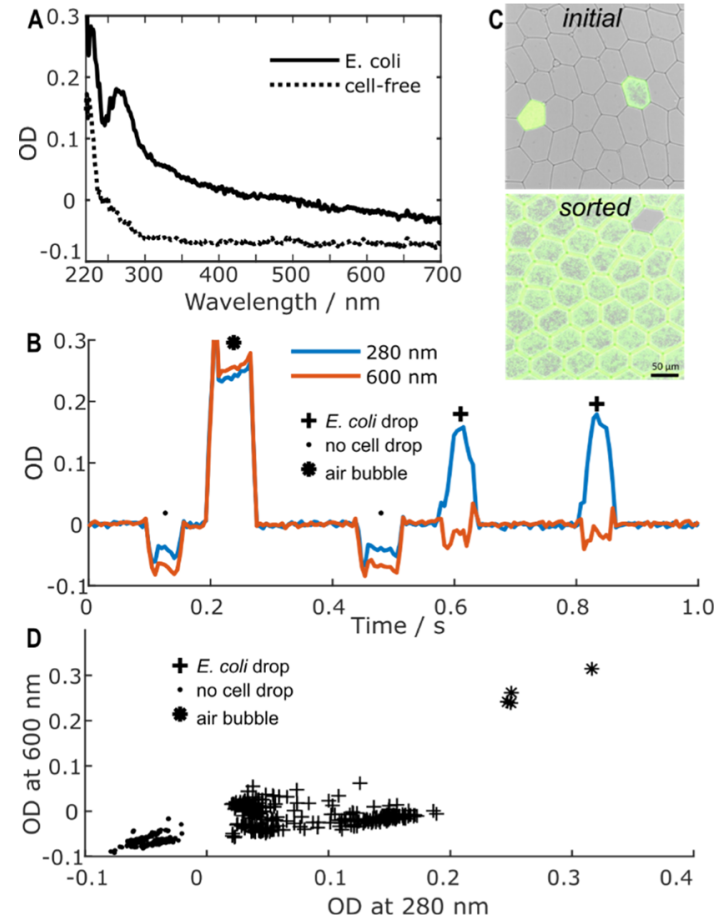

Figure 4. In-droplet UV-vis spectroscopy tracks cell growth. (A) Direct in-droplet UV-vis absorbance spectra using a $5 \mathrm{~ms}$ integration time. (B) In-droplet cell growth was tracked by UV-vis spectroscopy and distinguished from contaminants in the sample (such as air bubbles). (C) Combination of bright-field and fluorescence image of droplets before and after sorting. (D) Scatter plot of identified UVvis events for the $\mathrm{OD}$ at 280 and $600 \mathrm{~nm}$. For subfigures $\mathrm{B}$ and $\mathrm{D}$, the symbol "*” denotes air bubble, "+" denotes a droplet containing $E$. coli, and "." denotes a droplet with no cells.

ance droplet sorters demonstrated UVA measurements (at 365 $\mathrm{nm})^{8}$ but were not compatible with multiwavelength UVB $(280-315 \mathrm{~nm})$ and UVC $(<280 \mathrm{~nm})$ measurements required to monitor the ergothionase reaction. 
Here, we demonstrate the use of UVADS for label-free enzyme measurements. We performed an enzymatic assay on ergothionase activity for single $E$. coli cell-seeded microcolonies in droplets. We used E. coli BL21 (DE3) strains that produce ergothionase wild-type or one of the three ergothionase variants with $10^{3}$-fold less activity. The previously published mutants contain amino acid point mutations to the active site of the enzyme (Y54F, K384M, and E412Q). ${ }^{33}$ Cells were diluted into Mueller-Hinton cell media containing $1 \mathrm{mM}$ IPTG inducer, and the $5 \mathrm{mM}$ ergothioneine was isolated in droplets using a standard flow-focusing droplet generation device. The high cell dilution ensured that the majority of droplets were empty or contained single cells. The generated droplets were collected and placed at $37^{\circ} \mathrm{C}$ for $12 \mathrm{~h}$. During this time, single cells grew into monoclonal microcolonies while isolated in droplets. Microcolonies that produced ergothionase reacted with the ergothioneine substrate to produce thiourocanic acid.

After incubation, droplets were injected into the UVADS platform for label-free analysis of the reaction in Figure 5. UV-
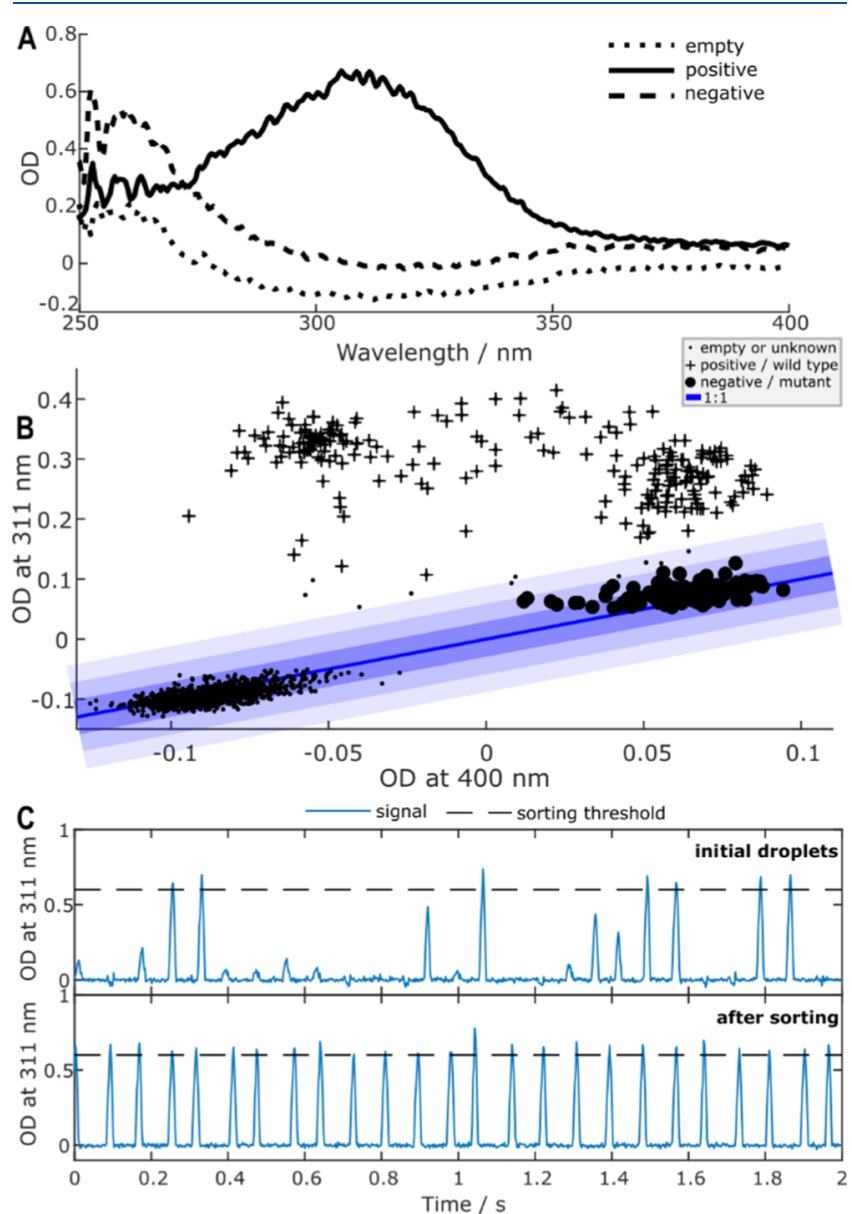

Figure 5. Label-free ergothionase activity assay of monoclonal microcolonies. (A) UV-vis spectra of empty droplets, negative (microcolony with no ergothionase activity), and positive (microcolony with wild-type ergothionase activity) are shown. (B) 2D scatter plot of droplets that are identified as empty or unknown ".", ergothionase positive " + ", and ergothionase negative "๑". A line is drawn along the 1:1 ratio to indicate the light-scattering baseline, and the shaded gradations are at 0.1 OD intervals. (C) Time course plots of OD $311 \mathrm{~nm}$ of droplets before and after sorting at a $0.6 \mathrm{OD}$ threshold. vis spectra from droplets generated separately containing either wild-type ergothionase or the mutants are shown in Figure 5A. As expected from the presence of a microcolony, a baseline shift in the visible-range OD is observed in both wild-type and mutant droplets caused by light scattering. The absorbance shift is more pronounced and not uniform in the UVC spectral range. This is due to the production of nucleic acids, proteins, and lipids associated with cell growth, as well as the consumption of ergothioneine in wild-type microcolonies. This highlights the potential challenges of making UV-vis measurement within a complex biological sample for small spectral features within the UVC spectral range. In this case, thiourocanic acid has a pronounced peak at $311 \mathrm{~nm}$ and the ergothionase-active wild-type microcolonies are easily observed.

In Figure 5B, a scatter plot of $400 \mathrm{~nm}$ OD by $311 \mathrm{~nm}$ OD of droplets is shown from a mixed population of droplets. To distinguish the ergothionase activity from generic light scattering resulting from cell growth, we used the proportional $311 \mathrm{~nm}$ OD to $400 \mathrm{~nm}$ OD shift as a baseline for selecting active droplets - as seen by the 1:1 line in Figure 5, allowing for facile identification of empty, enzymatically inactive microcolonies and those actively producing thiourocanic acid. By UVADS sorting, we set a threshold that can select droplets containing highly active ergothionase activity for further analysis or downstream applications. Figure 5C demonstrates the in-droplet UV-vis signal at $311 \mathrm{~nm}$ before and after sorting.

\section{CONCLUSIONS}

In this work, we introduced UVADS, a versatile tool for fullspectrum UV-vis droplet analysis and sorting. Our platform scales down the common UV-vis spectroscopy microtiterplate analytics to directly assess the chemical contents of subnanoliter droplets. Furthermore, we developed the open-source software application, SpectraSorter, for real-time spectral acquisition, analysis, and sorting. The available software application should allow this technology to be readily adapted by other laboratories. Using it, we could directly assess cell growth and enzyme activity label-free at high-throughput indroplets and collect droplets of interest. While the differentiation of intracellular compounds is difficult with absorption measurements, the system is particularly well-suited for measurements, where the analyte of interest is secreted out of the cell or the enzymatic reaction occurs within the droplet. Going forward, UVADS will be a powerful tool for label-free screening applications in droplets such as directed evolution, drug testing and discovery, and chemical synthesis (e.g., combinatorial chemistry or catalysis).

\section{ASSOCIATED CONTENT}

\section{SI Supporting Information}

The Supporting Information is available free of charge at https://pubs.acs.org/doi/10.1021/acs.analchem.1c02822.

Experimental information on the SU8 mold fabrication method, two-layer PDMS device assembly, droplet generation method, details about image acquisition, optimization of the UV-vis detection region, and montage of droplet sorting (PDF) 


\section{AUTHOR INFORMATION}

\section{Corresponding Author}

Petra S. Dittrich - Department of Biosystems Science and Engineering, ETH Zurich, 4058 Basel, Switzerland; NCCR Molecular Systems Engineering, 4058 Basel, Switzerland; (1) orcid.org/0000-0001-5359-8403; Email: petra.dittrich@ bsse.ethz.ch

\section{Authors}

Todd A. Duncombe - Department of Biosystems Science and Engineering, ETH Zurich, 4058 Basel, Switzerland; NCCR Molecular Systems Engineering, 4058 Basel, Switzerland; (1) orcid.org/0000-0002-2568-1709

Aaron Ponti - Department of Biosystems Science and Engineering, ETH Zurich, 4058 Basel, Switzerland

Florian P. Seebeck - NCCR Molecular Systems Engineering, 4058 Basel, Switzerland; Department of Chemistry, University of Basel, 4002 Basel, Switzerland; 이이.org/ 0000-0003-4625-1369

Complete contact information is available at:

https://pubs.acs.org/10.1021/acs.analchem.1c02822

\section{Notes}

The authors declare no competing financial interest.

\section{ACKNOWLEDGMENTS}

We thank Dr. Oliver Lischtschenko from Ocean Insight for assistance in the high-speed communication with the spectrophotometer. We thank Dr. Alice Maurer for providing us with the ergothionase mutants in E. coli. This work is supported by funding from the NCCR Molecular Systems Engineering, project no. 51NF40-182895.

\section{REFERENCES}

(1) Duncombe, T. A.; Tentori, A. M.; Herr, A. E. Nat. Rev. Mol. Cell Biol. 2015, 16, 554-567.

(2) Xi, H.-D.; et al. Lab Chip 2017, 17, 751-771.

(3) Schütz, S. S.; Beneyton, T.; Baret, J.-C.; Schneider, T. M. Lab Chip 2019, 19, 2220-2232.

(4) Isozaki, A.; et al. Sci. Adv. 2020, 6, No. eaba6712.

(5) Nitta, N.; et al. Cell 2018, 175, 266-276.

(6) Wang, X.; et al. Sci. Adv. 2020, 6, No. eabb3521.

(7) Agresti, J. J.; et al. Proc. Natl. Acad. Sci. U. S. A. 2010, 107, 40044009.

(8) Gielen, F.; et al. Proc. Natl. Acad. Sci. U. S. A. 2016, 113, No. E7383.

(9) Stucki, A.; Vallapurackal, J.; Ward, T. R.; Dittrich, P. S. Angew. Chem., Int. Ed. 2021, 60, 2-22, DOI: 10.1002/anie.202016154.

(10) Debs, B. E.; Utharala, R.; Balyasnikova, I. V.; Griffiths, A. D.; Merten, C. A. Proc. Natl. Acad. Sci. U. S. A. 2012, 109, 11570-11575.

(11) Vallejo, D.; Nikoomanzar, A.; Paegel, B. M.; Chaput, J. C. ACS Synth. Biol. 2019, 8, 1430-1440.

(12) Nan, L.; et al. Small 2020, 16, 1-11.

(13) Matuła, K.; Rivello, F.; Huck, W. T. S. Adv. Biosyst. 2020, 4, 1900188.

(14) Li, M.; Liu, H.; Zhuang, S.; Goda, K. RSC Adv. 2021, 11, 20944-20960.

(15) Rajauria, S.; Axline, C.; Gottstein, C.; Cleland, A. N. Nano Lett.

$\mathbf{2 0 1 5}, 15,469-475$.

(16) Hasan, S.; et al. Lab Chip 2019, 19, 403-409.

(17) Sesen, M.; Whyte, G. Sci. Rep. 2020, 10, 8736

(18) Honrado, C.; Bisegna, P.; Swami, N. S.; Caselli, F. Lab Chip

2021, 21, 22-54.

(19) Pacocha, N.; et al. Anal. Chem. 2021, 93, 843-850.

(20) Liu, W.-w.; Zhu, Y. Anal. Chim. Acta 2020, 1113, 66-84.
(21) Wang, X.; et al. Anal. Chem. 2017, 89, 12569-12577.

(22) Holland-Moritz, D. A.; et al. Angew. Chem., Int. Ed. 2020, 59, $4470-4477$.

(23) Choi, K.; Mudrik, J. M.; Wheeler, A. R. Anal. Bioanal. Chem. 2015, 407, 7467-7475.

(24) Duarte, L. C.; Figueredo, F.; Ribeiro, L. E. B.; Cortón, E.; Coltro, W. K. T. Anal. Chim. Acta 2019, 1071, 36-43.

(25) Gala De Pablo, J.; Lindley, M.; Hiramatsu, K.; Goda, K. Acc. Chem. Res. 2021, 54, 2132-2143.

(26) Küster, S. K.; et al. Anal. Chem. 2013, 85, 1285-1289.

(27) Verboket, P. E.; Borovinskaya, O.; Meyer, N.; Günther, D.; Dittrich, P. S. Anal. Chem. 2014, 86, 6012-6018.

(28) Petersen, N. J.; Mogensen, K. B.; Kutter, J. P. Electrophoresis 2002, 23, 3528-3536.

(29) Probst, J.; Howes, P.; Arosio, P.; Stavrakis, S.; DeMello, A. Anal. Chem. 2021, 93, 7673-7681.

(30) Yue, J.; Falke, F. H.; Schouten, J. C.; Nijhuis, T. A. Lab Chip 2013, 13, 4855-4863.

(31) Duncombe, T. A.; Ponti, A.; Dittrich, P. S. SpectraSorter: Ocean Insight Spectrophotometer Control Software for HighThroughput UV-VIS Analysis and Triggering. https://github.com/ SpectraSorter/SpectraSorter (accessed in 2021).

(32) Jusková, P.; et al. ACS Appl. Mater. Interfaces 2019, 11, 3469834706.

(33) Maurer, A.; Leisinger, F.; Lim, D.; Seebeck, F. P. Chem.-Eur. J. 2019, 25, 10298-10303.

(34) Cheah, I. K.; Halliwell, B. Antioxidants 2020, 9, 595.

(35) Borodina, I.; et al. Nutr. Res. Rev. 2020, 33, 190-217. 